\title{
Genu of the Corpus Callosum
}

National Cancer Institute

\section{Source}

National Cancer Institute. Genu of the Corpus Callosum. NCI Thesaurus. Code C32675.

The anterior portion of the corpus callosum that bends downward and backward. 